\title{
Hyers-Ulam Stability of Third Order Euler's Differential Equations
}

\author{
A. K. Tripathy and A. Satapathy \\ Department of Mathematics, Sambalpur University, Sambalpur 768019, India \\ Correspondence should be addressed to A. K. Tripathy; arun_tripathy70@rediffmail.com
}

Received 1 May 2014; Accepted 8 October 2014; Published 4 November 2014

Academic Editor: Ivo Petras

Copyright (C) 2014 A. K. Tripathy and A. Satapathy. This is an open access article distributed under the Creative Commons Attribution License, which permits unrestricted use, distribution, and reproduction in any medium, provided the original work is properly cited.

We investigate the Hyers-Ulam stability of third order Euler's differential equations of the form $t^{3} y^{\prime \prime \prime}+\alpha t^{2} y^{\prime \prime}+\beta t y^{\prime}+\gamma y=0$ on any open interval $I=(a, b), 0<a<b \leq \infty$ or $-\infty<a<b<0$, where $\alpha, \beta$, and $\gamma$ are complex constants.

\section{Introduction}

In 1940, Ulam gave a wide ranging talk before the Mathematics Club of the University of Wisconsin in which he discussed a number of important unsolved problems [1]. Among such problems is a problem concerning the stability of functional equations: give conditions in order for a linear function near an approximately linear function to exist.

In the following year, Hyers [2] gave an answer to the problem of Ulam for additive functions defined on Banach spaces.

Let $X_{1}$ and $X_{2}$ be two real Banach spaces and let $\epsilon>0$. Then, for every function $f: X \rightarrow Y$ satisfying

$$
\|f(x+y)-f(x)-f(y)\| \leq \epsilon, \quad\left(x, y \in X_{1}\right)
$$

there exists a unique additive function $A: X_{1} \rightarrow X_{2}$ with the property

$$
\|f(x)-A(x)\| \leq \epsilon, \quad x \in X_{1} .
$$

Furthermore, the result of Hyers has been generalized by Rassias [3, 4]. Since then, the stability problems of various functional equations have been investigated by many authors (see, e.g., [1,5-8]). A generalization of Ulam's problem was recently proposed by replacing functional equations with differential equations.

The differential equation

$$
\phi\left(t, y, y^{\prime}, \ldots, y^{(n)}\right)=0
$$

has Hyers-Ulam stability; if for given $\epsilon>0$ and a function $y$ such that

$$
\phi\left|\left(t, y, y^{\prime}, \ldots, y^{(n)}\right)\right| \leq \epsilon,
$$

then there exists a solution $y_{a}$ of the differential equation such that $\left|y(t)-y_{a}(t)\right| \leq K(\epsilon)$ and $\lim _{\epsilon \rightarrow 0} K(\epsilon)=0$.

If the preceding statement is also true when we replace $\epsilon$ and $K(\epsilon)$ by $\phi(t)$ and $\psi(t)$, respectively, where $\phi$ and $\psi$ are appropriate functions not depending on $y$ and $y_{a}$ explicitly, then we say that the corresponding differential equation has the generalized Hyers-Ulam stability.

Obloza seems to be the first author who has investigated the Hyers-Ulam stability of linear differential equations (see, e.g., $[9,10])$. Thereafter, Alsina and Ger published their work [11], which handles the Hyers-Ulam stability of the linear differential equation $y^{\prime}(t)=y(t)$.

If a differentiable function $y(t)$ is a solution of the inequality $\left|y^{\prime}(t)-y(t)\right| \leq \epsilon$ for any $t \in(a, \infty)$, then there exists a constant $c$ such that $\left|y^{\prime}(t)-c e^{t}\right| \leq 3 \epsilon$, for all $t \epsilon$ $(a, \infty)$.

In [1], Rezaei et al. have discussed the Hyers-Ulam stability of linear differential equations of first and $n$th order by applying Laplace transform which is comparable with the other methods available in the literature.

It is Jung et al. who have investigated the Hyers-Ulam stability of linear differential equations of different classes including the stability of the delay differential equation 
$y^{\prime}(t)=\lambda y(t-\tau)$, where $\tau>0$ is a constant (see, e.g., [5$7,12-14])$. Among the works, we are motivated by the results of [13], where he has studied the Hyers-Ulam stability of the following Euler's differential equations:

$$
\begin{gathered}
t y^{\prime}(t)+\alpha y(t)+\beta t^{r} x_{0}=0, \\
t^{2} y^{\prime \prime}(t)+\alpha t y^{\prime}(t)+\beta y(t)=0,
\end{gathered}
$$

where $\alpha, \beta$, and $\gamma$ are complex constants. We may note that the Hyers-Ulam stability of (6) depends on the Hyers-Ulam stability of (5) for every fixed $x_{0} \neq 0$. We mentioned here the important results of [13].

Theorem 1. Let $X$ be a complex Banach space and let $I=(a, b)$ be an open interval such that $0<a<b \leq \infty$ or $-\infty<a<b<$ 0 . Assume that a function $\phi: I \rightarrow[0, \infty)$ is given, that $\alpha, \beta$, and $\gamma$ are complex constants, and that $x_{0}$ is a fixed element of $X$. Furthermore, suppose a continuously differentiable function $f: I \rightarrow X$ satisfies

$$
\left\|t y^{\prime}(t)+\alpha y(t)+\beta t^{r} x_{0}\right\| \leq \phi(t), \quad t \in I
$$

If both $t^{\alpha+r-1}$ and $t^{\alpha-1} \phi(t)$ are integrable on $(a, c)$, for any $c$ with $a<c \leq b$, then there exists a unique solution $f_{0}: I \rightarrow X$ of the differential equation (5) such that

$$
\left\|f(t)-f_{0}(t)\right\| \leq\left|t^{-\alpha}\right|\left|\int_{t}^{b} v^{\alpha-1} \phi(v) d v\right|
$$

for any $t \in I$.

Indeed, the unique solution of (5) in the complex Banach space $X$ is given by

$$
f_{0}(t)= \begin{cases}\left(\frac{a}{t}\right)^{\alpha} x-\frac{\beta}{\alpha+r} \frac{1}{t^{\alpha}}\left(t^{\alpha+r}-a^{\alpha+r}\right) x_{0}, & (\alpha+r) \neq 0, \\ \left(\frac{a}{t}\right)^{\alpha} x-\frac{\beta}{t^{\alpha}}(\ln |t|-\ln |a|) x_{0} . & (\alpha+r)=0 .\end{cases}
$$

Theorem 2. If a twice continuously differentiable function $f$ : $I \rightarrow R$ satisfies the differential inequality

$$
\left|t^{2} f^{\prime \prime}(t)+\alpha t f^{\prime}(t)+\beta f(t)\right| \leq \epsilon
$$

for all $t \epsilon I$ and for some $\epsilon>0$, then there exists a solution $f_{0}: I \rightarrow R$ of (6) such that

$$
\left|f(t)-f_{0}(t)\right| \leq \frac{\epsilon}{|\beta|}\left|\left(\frac{b}{t}\right)^{c}-1\right|
$$

for any $t \in I$, where $c$ is a negative real number. In particular, if $I=(a, \infty), a>0$, then

$$
\left|f(t)-f_{0}(t)\right| \leq \frac{\epsilon}{|\beta|}, \quad \forall t>a .
$$

In this theorem, we may note that the unique solution of (6) is given by

$$
f_{0}(t)=\left(c_{3}+\frac{c_{0}}{d(c-d)}\right)\left(\frac{a}{t}\right)^{c}-\frac{c_{0}}{d(c-d)}\left(\frac{a}{t}\right)^{d},
$$

where

$$
\begin{aligned}
& c=\frac{(\alpha-1)-\left[(1-\alpha)^{2}-4 \beta\right]^{1 / 2}}{2}, \\
& d=\frac{(\alpha-1)+\left[(1-\alpha)^{2}-4 \beta\right]^{1 / 2}}{2}
\end{aligned}
$$

are real constants and $c_{3}$ is any arbitrary constant.

Theorem 3. Let $X$ be a complex Banach space and let $I=$ $(a, b)$ be an open interval such that $0<a<b \leq \infty$ or $-\infty<$ $a<b<0$. Assume that a function $\phi: I \rightarrow[0, \infty)$ is given and that $h: I \rightarrow X$ is a continuous function. Furthermore, suppose a continuously differentiable function $f: I \rightarrow X$ satisfies

$$
\left\|t y^{\prime}(t)+\alpha y(t)+h(t)\right\| \leq \phi(t), \quad t \in I .
$$

If both $t^{\alpha-1} \phi(t)$ and $t^{\alpha-1} h(t)$ are integrable on $(a, c)$, for any $c$ with $a<c \leq b$, then there exists a unique solution $f_{0}: I \rightarrow X$ of the differential equation

$$
t y^{\prime}(t)+\alpha y(t)+h(t)=0
$$

such that (8) holds for any $t \in I$, where $\alpha$ is a complex constant.

Proof. The proof of the theorem follows from the proof of Theorem 1, if we set

$$
z(t)=\left(\frac{t}{a}\right)^{\alpha} f(t)+\int_{a}^{t} \frac{u^{\alpha-1}}{a^{\alpha}} h(u) d u,
$$

where the unique solution is given by

$$
f_{0}(t)=\left(\frac{a}{t}\right)^{\alpha}-\frac{1}{t^{\alpha}} \int_{a}^{t} u^{\alpha-1} h(u) d u .
$$

The details are omitted.

Remark 4. We may note that (5) is a particular case of (16). Hence, Theorems 1 and 3 are comparable.

The objective of this work is to investigate the generalized Hyers-Ulam stability of the following Euler's differential equations of the form

$$
\begin{gathered}
t^{2} y^{\prime \prime}(t)+\alpha t y^{\prime}(t)+\beta y(t)+\gamma t^{r} x_{0}=0, \\
t^{3} y^{\prime \prime \prime}(t)+\alpha t^{2} y^{\prime \prime}(t)+\beta t y^{\prime}(t)+\gamma y(t)=0,
\end{gathered}
$$

where $\alpha, \beta, \gamma$, and $r$ are complex constants. Intuitively, we shall prove that if a twice continuously differentiable function $f: I \rightarrow X$ satisfies the differential inequality

$$
\left\|t^{2} y^{\prime \prime}(t)+\alpha t y^{\prime}(t)+\beta y(t)+\gamma t^{r} x_{0}\right\| \leq \phi(t)
$$

for all $t \in I$, where $x_{0}$ is a fixed element of the complex Banach space $X$ and $I=(a, b)$ with $0<a<b \leq \infty$ or $-\infty<a<$ $b<0$, then there exists a twice continuously differentiable solution $f_{0}(t)$ of $(19)$ such that

$$
\left\|f(t)-f_{0}(t)\right\| \leq\left|t^{l}\right|\left|\int_{t}^{b} u^{-l-1} \psi(u) d u\right|
$$


for any $t \in I$, where

$$
\psi(t)=\left|t^{m}\right|\left|\int_{t}^{b} u^{-m-1} \phi(u) d u\right| .
$$

We also apply this result to the investigation of the HyersUlam stability of (20).

\section{Hyers-Ulam Stability of (19)}

Throughout this section, we let $I=(a, b)$ with $0<a<b \leq \infty$ or $-\infty<a<b<0$. For fixed $x_{0} \neq 0$, the general solution of (19) in the class of real valued functions defined on $I$ is given by

$$
y(t)=\left\{\begin{array}{l}
c_{1} t^{l}+c_{2} t^{m}-\frac{\gamma x_{0} t^{r}}{g(r)}, \\
g(r) \neq 0, \quad l \neq m, \\
c_{1} t^{l}+c_{2} t^{m}-\frac{\gamma x_{0} t^{r} \ln |t|}{g^{\prime}(r)}, \\
g(r)=0 \neq g^{\prime}(r), \quad l \neq m, \\
\left(c_{1}+c_{2} \ln |t|\right) t^{l}-\frac{\gamma x_{0} t^{r}}{g(r)}, \\
g(r) \neq 0, \quad l=m, \\
y(t)=\left(c_{1}+c_{2} \ln |t|\right) t^{l}-\frac{\gamma x_{0} t^{r}(\ln |t|)^{2}}{2}, \\
g(r)=0=g^{\prime}(r), \quad l=m,
\end{array}\right.
$$

where $g(r)=r^{2}+(\alpha-1) r+\beta$ and $c_{1}, c_{2}$ are arbitrary constants.

Remark 5. Indeed, $g^{\prime}(r)=2 r+\alpha-1$. If $g(r)=0$, then either $r-l=0$ or $r-m=0$. Therefore, $r-l=0$ implies that $2 r+\alpha-1=2 r-l-m=r-m \neq 0$ and $r-m=0$ implies that $2 r+\alpha-1=r-l \neq 0$. Hence, the second solution could be any one of the following:

$$
\begin{array}{r}
c_{1} t^{l}+c_{2} t^{m}-\frac{\gamma x_{0} t^{r} \ln |t|}{r-m} ; \quad r-l=0, \\
r-m \neq 0, \quad l \neq m, \\
c_{1} t^{l}+c_{2} t^{m}-\frac{\gamma x_{0} t^{r} \ln |t|}{r-l} ; \quad r-l \neq 0, \\
r-m=0, \quad l \neq m .
\end{array}
$$

Theorem 6. Let $X$ be a complex Banach space and let $I=$ $(a, b)$ be an open interval with either $0<a<b \leq \infty$ or $-\infty<a<b<0$. Assume that a function $\phi: I \rightarrow[0, \infty)$ is given, that $\alpha, \beta, \gamma$, and $r$ are complex constants, and that $x_{0}$ is a fixed element of $X$. Furthermore, suppose a twice continuously differentiable function $f: I \rightarrow X$ satisfies the differential inequality (21). If $t^{r-l-1}, t^{r-m-1}, t^{m-l-1}, t^{-m-1} \phi(t)$, and $t^{-l-1} \psi(t)$ are integrable on $(a, c)$, for any $c$ with $a<c \leq b$, then there exists a unique solution $f_{0}: I \rightarrow X$ (which is twice continuously differentiable) of (19) such that (22) holds for any $t \in I$, where $l$ and $m$ are the roots of $g(r)=0$.
Proof. We prove the theorem for five possible cases; namely,
(i) $(r-l)(r-m) \neq 0, l \neq m$;
(ii) $r-l=0 \neq r-m, l \neq m$;
(iii) $r-l \neq 0=r-m, l \neq m$;
(iv) $r-l \neq 0, l=m$;
(v) $r-l=0, l=m$.

Case (i). Suppose that $X$ is a complex Banach space and $f: I \rightarrow X$ is a twice continuously differentiable function satisfying the differential inequality (21). Define $h: I \rightarrow X$ such that $h(t)=t f^{\prime}(t)-l f(t)$. Then it is easy to verify that

$$
\begin{aligned}
& \left\|t h^{\prime}(t)-m h(t)+\gamma t^{r} x_{0}\right\| \\
& =\left\|t^{2} f^{\prime \prime}(t)+(1-l-m) t f^{\prime}(t)+\operatorname{lm} f(t)+\gamma t^{r} x_{0}\right\| \\
& =\left\|t^{2} f^{\prime \prime}(t)+\alpha t f^{\prime}(t)+\beta f(t)+\gamma t^{r} x_{0}\right\| \\
& \quad \leq \phi(t) .
\end{aligned}
$$

Hence, by Theorem 1 , it follows that there exists a unique solution $h_{0}: I \rightarrow X$ of the differential equation $t y^{\prime}-m y+$ $\gamma t^{r} x_{0}=0$ such that

$$
\left\|h(t)-h_{0}(t)\right\| \leq\left|t^{m}\right|\left|\int_{t}^{b} v^{-m-1} \phi(v) d v\right|
$$

provided that $t^{r-m-1}$ and $t^{-m-1} \phi(t)$ are integrable on $(a, c)$, for any $c$ with $a<c \leq b$. Indeed,

$$
h_{0}(t)=\left(\frac{a}{t}\right)^{-m} x-\frac{\gamma t^{m}}{r-m}\left(t^{r-m}-a^{r-m}\right) x_{0}
$$

where $x$ is a limit point in $X$. If we denote

$$
\psi(t)=\left|t^{m} \int_{t}^{b} v^{-m-1} \phi(v) d v\right|,
$$

then (27) becomes

$$
\left\|t f^{\prime}(t)-l f(t)-h_{0}(t)\right\| \leq \psi(t) .
$$

Clearly, $\psi: I \rightarrow[0, \infty)$. By Theorem 3 , there exists a unique solution $f_{1}: I \rightarrow X$ of the differential equation $t y^{\prime}(t)-l y(t)-$ $h_{0}(t)=0$ such that

$$
\left\|f(t)-f_{1}(t)\right\| \leq\left|t^{l}\right|\left|\int_{t}^{b} u^{-l-1} \psi(u) d u\right|
$$

provided that $t^{-l-1} \psi(t)$ and $t^{-l-1} h_{0}(t)$ are integrable on $(a, c)$, for any $c$ with $a<c \leq b$. Clearly, $t^{-l-1} h_{0}(t)$ is integrable if and only if $t^{m-l-1}$ and $t^{r-l-1}$ are integrable. According to Theorem 3,

$$
f_{1}(t)=\left(\frac{t}{a}\right)^{l} x_{1}+t^{l} \int_{a}^{t} u^{-l-1} h_{0}(u) d u,
$$


where $x_{1}$ is limit point in $X$. It is easy to verify that

$$
\begin{aligned}
\int_{a}^{t} u^{-l-1} h_{0}(u) d u= & {\left[\frac{x}{a^{m}}+\frac{\gamma x_{0} a^{r-m}}{r-m}\right] \frac{t^{m-l}}{m-l} } \\
& -\frac{x}{a^{l}(m-l)}-\frac{\gamma x_{0} t^{r-l}}{(r-l)(r-m)} \\
& -\frac{\gamma x_{0} a^{r-l}}{(r-l)(m-l)} .
\end{aligned}
$$

Consequently,

$$
\begin{aligned}
f_{1}(t)= & {\left[\frac{x}{a^{l}}-\frac{\gamma x_{0} a^{r-l}}{(r-l)(m-l)}-\frac{x}{a^{l}(m-l)}\right] t^{l} } \\
& +\left[\frac{x}{a^{m}(m-l)}+\frac{\gamma x_{0} a^{r-m}}{(r-m)(m-l)}\right] t^{m}-\frac{\gamma x_{0} t^{r}}{g(r)}
\end{aligned}
$$

which is a solution of (19).

Case (ii). Proceeding as in Case (i), we find a unique solution $f_{2}: I \rightarrow X$ of the equation $t y^{\prime}(t)-l y(t)-h_{0}(t)=0$ such that

$$
\begin{gathered}
\left\|f(t)-f_{2}(t)\right\| \leq\left|t^{l}\right|\left|\int_{t}^{b} u^{-l-1} \psi(u) d u\right|, \\
f_{2}(t)=\left(\frac{t}{a}\right)^{l} x_{2}+t^{l} \int_{a}^{t} u^{-l-1} h_{0}(u) d u,
\end{gathered}
$$

where $x_{2}$ is a limit point in $X$. Clearly,

$$
\begin{aligned}
& \int_{a}^{t} u^{-l-1} h_{0}(u) d u \\
&=\int_{a}^{t}\left[\frac{x}{a^{m}} u^{m-l-1}-\frac{\gamma x_{0} u^{-1}}{r-m}+\frac{\gamma x_{0} a^{r-m}}{r-m} u^{m-l-1}\right] d u \\
&= {\left[\frac{x}{a^{m}}+\frac{\gamma x_{0} a^{r-m}}{r-m}\right] \frac{t^{m-l}}{m-l}+\frac{\gamma x_{0}}{(r-m)(l-m)} } \\
&-\frac{x}{a^{l}(m-l)}-\frac{\gamma x_{0}}{(r-m)} \ln \left|\frac{t}{a}\right|
\end{aligned}
$$

implies that

$$
\begin{aligned}
f_{2}(t)= & {\left[\frac{x_{2}}{a^{l}}+\frac{\gamma x_{0}}{(r-m)^{2}}+\frac{x}{a^{l}(r-m)}\right] t^{l} } \\
& +\left[\frac{x}{a^{m}(m-l)}-\frac{\gamma x_{0} a^{r-m}}{(r-m)^{2}}\right] t^{m}-\frac{\gamma x_{0} t^{r}}{(r-m)} \ln \left|\frac{\mathrm{t}}{a}\right|
\end{aligned}
$$

which is a solution of (19).

Case (iii). Interchanging the roles of $l$ and $m$ in Case (ii), we find the unique solution $f_{3}(t)$ as

$$
\begin{aligned}
f_{3}(t)= & {\left[\frac{x_{3}}{a^{l}}-\frac{x}{a^{l}(m-l)}-\frac{\gamma x_{0} a^{m-l}}{(m-l)^{2}}\right] t^{l} } \\
& +\left[\frac{x}{a^{m}(m-l)}+\frac{\gamma x_{0}}{(m-l)^{2}}\right] t^{m}-\frac{\gamma x_{0} t^{r}}{(r-l)} \ln \left|\frac{t}{a}\right|,
\end{aligned}
$$

where $x_{3} \in X$.
Case (iv). In this case, we proceed as in Case (i). Indeed, for $l=m$,

$$
\begin{aligned}
\int_{a}^{t} u^{-l-1} h_{0}(u) d u= & {\left[\frac{x}{a^{l}}+\frac{\gamma x_{0} a^{r-l}}{r-l}\right] \ln \left|\frac{t}{a}\right| } \\
& +\frac{\gamma x_{0}}{(r-l)^{2}}\left[a^{r-l}-t^{r-l}\right]
\end{aligned}
$$

and the unique solution $f_{4}(t)$ is given by

$$
\begin{aligned}
f_{4}(t)= & {\left[\left\{\frac{x_{4}}{a^{l}}+\frac{\gamma x_{0} a^{r-l}}{(r-l)^{2}}\right\}+\left\{\frac{x}{a^{l}}+\frac{\gamma x_{0} a^{r-l}}{(r-l)}\right\} \ln \left|\frac{t}{a}\right|\right] t^{l} } \\
& -\frac{\gamma x_{0} t^{r}}{(r-l)^{2}}
\end{aligned}
$$

where $x_{4} \in X$.

Case (v). In this case,

$$
h_{0}(t)=\left(\frac{t}{a}\right)^{l} x-\gamma x_{0} t^{l} \ln \left|\frac{t}{a}\right| .
$$

Using the same type of argument as in Case (i), we find the unique solution $f_{5}(t)$ as

$$
\begin{aligned}
f_{5}(t) & =\left(\frac{t}{a}\right)^{l} x_{5}+t^{l} \int_{a}^{t} u^{-l-1} h_{0}(u) d u \\
& =\left(\frac{t}{a}\right)^{l} x_{5}+\frac{x}{a^{l}} t^{l} \ln \left|\frac{t}{a}\right|-\frac{\gamma x_{0}}{2} t^{l}\left(\ln \left|\frac{t}{a}\right|\right)^{2} \\
& =\left[\frac{x_{5}}{a^{l}}+\frac{x}{a^{l}} \ln \left|\frac{t}{a}\right|\right] t^{l}-\frac{\gamma x_{0}}{2} t^{r}\left(\ln \left|\frac{t}{a}\right|\right)^{2},
\end{aligned}
$$

where $x_{5} \in X$. This completes the proof of the theorem.

\section{Hyers-Ulam Stability of (20)}

In this section, we shall investigate the Hyers-Ulam stability of (20) on any open interval $I=(a, b)$ with either $0<a<b \leq$ $\infty$ or $-\infty<a<b<0$. We may note that $\alpha=3-(l+m+n)$, $\beta=l m+m n+n l-l-m-n+1$, and $\gamma=-l m n$, when $l, m$, and $n$ are the characteristic roots of the associated characteristic equation of (20).

Theorem 7. Let $X$ be a complex Banach space. Assume that $\theta: I \rightarrow[0, \infty)$ is given. Furthermore, assume that $t^{n-l-1}$, $t^{n-m-1}, t^{m-l-1}, t^{-n-1} \theta(t), t^{-m-1} \lambda(t)$, and $t^{-l-1} \eta(t)$ are integrable on $(a, c)$ with $a<c \leq b$, where

$$
\begin{aligned}
& \lambda(t)=\left|t^{n} \int_{t}^{b} u^{-n-1} \theta(u) d u\right|, \\
& \eta(t)=\left|t^{m} \int_{t}^{b} v^{-m-1} \lambda(v) d v\right| .
\end{aligned}
$$

Suppose that $f \in C^{3}(I, X)$ and satisfies

$$
\left\|t^{3} f^{\prime \prime \prime}(t)+\alpha t^{2} f^{\prime \prime}(t)+\beta t f^{\prime}(t)+\gamma f(t)\right\| \leq \theta(t),
$$


for all $t \in I$. Then, there exists a unique solution $f_{0} \in C^{3}(I, X)$ of (20) such that

$$
\left\|f(t)-f_{0}(t)\right\| \leq\left|t^{l} \int_{t}^{b} s^{-l-1} \eta(s) d s\right|
$$

Proof. Let $f: I \rightarrow X$ be such that (44) holds, for $t \in I$. Define $w: I \rightarrow X$ such that

$$
w(t)=t^{2} f^{\prime \prime}(t)+(1-l-m) t f^{\prime}(t)+\operatorname{lm} f(t) .
$$

Indeed,

$$
\left\|t w^{\prime}(t)-n w(t)\right\| \leq \theta(t), \quad t \in I .
$$

Hence, using Theorem 3, it follows that there exists a unique $w_{0}: I \rightarrow X$ such that

$$
\left\|w(t)-w_{0}(t)\right\| \leq\left|t^{n}\right|\left|\int_{t}^{b} u^{-n-1} \theta(u) d u\right|=\lambda(t)
$$

where $w_{0}(t)=(t / a)^{n} x$ and $x$ is a limit point in $X$. Ultimately, (48) becomes

$$
\left\|t^{2} f^{\prime \prime}(t)+(1-l-m) t f^{\prime}(t)+\operatorname{lm} f(t)-a^{-n} t^{n} x\right\| \leq \lambda(t),
$$

Now, we can apply Theorem 6 to the above differential inequality for five possible cases. Consequently,

$$
\begin{aligned}
f_{1}(t)= & {\left[x_{1}-\frac{x_{2}}{m-l}+\frac{x}{(n-l)(n-m)}\right]\left(\frac{t}{a}\right)^{l} } \\
& +\left[\frac{x_{2}}{m-l}+\frac{x}{(n-m)(l-m)}\right]\left(\frac{t}{a}\right)^{m} \\
& +\frac{x}{(n-l)(n-m)}\left(\frac{t}{a}\right)^{n},
\end{aligned}
$$

$f_{2}(t)$

$$
\begin{aligned}
= & {\left[\frac{x_{3}}{a^{l}}+\frac{x_{2}}{a^{l}(n-m)}-\frac{x}{a^{n}(n-m)^{2}}+\frac{x}{a^{n}(n-m)} \ln \left|\frac{t}{a}\right|\right] t^{l} } \\
& +\left[\frac{x_{2}}{a^{m}(m-l)}+\frac{x}{a^{m}(n-m)^{2}}\right] t^{m},
\end{aligned}
$$$$
f_{3}(t)
$$$$
=\left[\frac{x_{4}}{a^{l}}-\frac{x_{2}}{a^{l}(m-l)}+\frac{x}{a^{l}(m-l)^{2}}\right] t^{l}
$$$$
+\left[\frac{x_{2}}{a^{m}(m-l)}-\frac{x}{a^{m}(m-l)^{2}}+\frac{x}{a^{m}(m-l)} \ln \left|\frac{t}{a}\right|\right] t^{m},
$$

$$
\begin{aligned}
& f_{4}(t) \\
&= {\left[\frac{x_{4}}{a^{l}}-\frac{x}{a^{l}(n-l)^{2}}+\left\{\frac{x_{2}}{a^{l}}-\frac{x}{a^{l}(n-l)}\right\} \ln \left|\frac{t}{a}\right|\right] t^{l} } \\
&+\frac{x}{(n-l)^{2}}\left(\frac{t}{a}\right)^{n}, \\
& f_{5}(t)=\left[\frac{x_{5}}{a^{l}}+\frac{x_{2}}{a^{l}} \ln \left|\frac{t}{a}\right|+\frac{x}{2 a^{n}}\left(\ln \left|\frac{t}{a}\right|\right)^{2}\right] t^{l}
\end{aligned}
$$

are the unique solutions with respect to the five possible cases, respectively. In fact, they are the possible solutions of (20). Hence, the theorem is proved.

Corollary 8. Let $X=\mathbb{R}$ be a real Banach space. Assume that $t^{n-l-1}, t^{n-m-1}$, and $t^{m-l-1}$ are integrable on $I=(a, b)$ with $0<$ $a<b \leq \infty$ or $-\infty<a<b<0$, where $l, m$, and $n$ are positive roots of the associated characteristic equation of (20). Suppose that $f \in C^{3}(I, X)$ and satisfies

$$
\left\|t^{3} f^{\prime \prime \prime}(t)+\alpha t^{2} f^{\prime \prime}(t)+\beta t f^{\prime}(t)+\gamma f(t)\right\| \leq \epsilon,
$$

for all $t \in I$. Then, there exists a unique solution $f_{0} \in C^{3}(I, X)$ of (20) such that

$$
\left\|f(t)-f_{0}(t)\right\| \leq \frac{|\epsilon|}{l m n} .
$$

Proof. Let $f: I \rightarrow X$ be such that (51) holds, for all $t \in I$. Since all the conditions of Theorem 7 are satisfied, then there exists a unique solution $f_{0}: I \rightarrow X$ of (20) such that

$$
\lambda(t)=\left|t^{n} \int_{t}^{b} u^{-n-1} \epsilon d u\right|=|\epsilon|\left|t^{n}\right|\left|\left[\frac{u^{-n}}{-n}\right]_{t}^{b}\right|=\left|\frac{\epsilon}{n}\right|
$$

when $I=(-\infty, \infty)$. Then, for $I=(-\infty, \infty)$,

$$
\eta(t)=\left|t^{m} \int_{t}^{b} v^{-m-1} \lambda(v) d v\right|=\left|\frac{\epsilon}{m n}\right|\left|\left(\frac{t}{b}\right)^{m}-1\right|=\left|\frac{\epsilon}{m n}\right| .
$$

Hence,

$$
\left\|f(t)-f_{0}(t)\right\| \leq\left|t^{l} \int_{t}^{b} s^{-l-1} \eta(s) d s\right|=\left|\frac{\epsilon}{l m n}\right|\left|\left(\frac{t}{b}\right)^{l}-1\right| .
$$

Consequently,

$$
\left\|f(t)-f_{0}(t)\right\| \leq \frac{|\epsilon|}{l m n} \text { as } b \longrightarrow \infty,
$$

where $f_{0}(t)$ can be chosen from Theorem 7 .

Example 9. Let $X=\mathbb{R}$ be a Banach space and let $I=(a, \infty)$, $a>0$. Consider Euler's equation

$$
t^{3} y^{\prime \prime \prime}(t)-6 t^{2} y^{\prime \prime}(t)+18 t y^{\prime}(t)-24 y(t)=0
$$


with $l=4, m=3$, and $n=2$. Suppose that $f: I \rightarrow X$ satisfies the differential inequality

$$
\left\|t^{3} f^{\prime \prime \prime}(t)-6 t^{2} f^{\prime \prime}(t)+18 t f^{\prime}(t)-24 f(t)\right\| \leq \epsilon,
$$

for any $t \in I$. By Corollary 8 , there exists a unique solution $f_{0}: I \rightarrow X$ of (57) such that

$$
\left\|f(t)-f_{0}(t)\right\| \leq \frac{\epsilon}{24}
$$

for any $t \in I$ and for unique $x, x_{1}, x_{2} \in X$,

$$
f_{0}(t)=\left\{\frac{x}{2}+x_{1}+x_{2}\right\}\left(\frac{t}{a}\right)^{4}+\left\{x-x_{2}\right\}\left(\frac{t}{a}\right)^{3}+\left(\frac{x}{2}\right)\left(\frac{t}{a}\right)^{2} .
$$

\section{Conflict of Interests}

The authors declare that there is no conflict of interests regarding the publication of this paper.

\section{Acknowledgment}

The authors are thankful to the referee for his helpful suggestions and necessary corrections in the completion of this paper.

\section{References}

[1] H. Rezaei, S.-M. Jung, and T. M. Rassias, "Laplace transform and Hyers-Ulam stability of linear differential equations," Journal of Mathematical Analysis and Applications, vol. 403, no. 1, pp. 244251, 2013.

[2] D. H. Hyers, "On the stability of the linear functional equations," Proceedings of the National Academy of Sciences of the United States of America, vol. 27, pp. 222-224, 1941.

[3] T. M. Rassias, "On the stability of linear mapping in Banach spaces," Proceedings of the American Mathematical Society, vol. 72, pp. 297-300, 1978.

[4] T. M. Rassias, "On the stability of functional equations and a problem of Ulam," Acta Applicandae Mathematicae, vol. 62, no. 1, pp. 23-30, 2000.

[5] S.-M. Jung and T. M. Rassias, "Ulam's problem for approximate homomorphisms in connection with Bernoulli's differential equation," Applied Mathematics and Computation, vol. 187, no. 1, pp. 223-227, 2007.

[6] S.-M. Jung and T. M. Rassias, "Generalized Hyers-Ulam stability of Riccati differential equation," Mathematical Inequalities and Applications, vol. 11, no. 4, pp. 777-782, 2008.

[7] S. M. Jung and J. Brzdek, "Hyers-Ulam stability of the delay differential equation $y^{\prime}(t)=\lambda y(t-\tau)$," Abstract and Applied Analysis, vol. 2010, Article ID 372176, 10 pages, 2010.

[8] Y. Li and Y. Shen, "Hyers-Ulam stability of linear differential equations of second order," Applied Mathematics Letters, vol. 23, no. 3, pp. 306-309, 2010.

[9] M. Obloza, "Hyers stability of the linear differential equation," Rocznik Naukowo-Dydaktyczny. Prace Matematyczne, vol. 13, pp. 259-270, 1993.
[10] M. Obloza, "Connections between Hyers and Lyapunov stability of the ordinary differential equations," Rocznik NaukowoDydaktyczny. Prace Matematyczne, vol. 14, pp. 141-146, 1997.

[11] C. Alsina and R. Ger, "On some inequalities and stability results related to the exponential function," Journal of Inequalities and Applications, vol. 2, pp. 373-380, 1998.

[12] S.-M. Jung, "Hyers-Ulam stability of linear differential equations of first order," Applied Mathematics Letters, vol. 17, no. 10, pp. 1135-1140, 2004.

[13] S.-M. Jung, "Hyers-Ulam stability of linear differential equations of first order, III," Journal of Mathematical Analysis and Applications, vol. 311, no. 1, pp. 139-146, 2005.

[14] S.-M. Jung, "Hyers-Ulam stability of linear differential equations of first order, II," Applied Mathematics Letters, vol. 19, no. 9, pp. 854-858, 2006. 

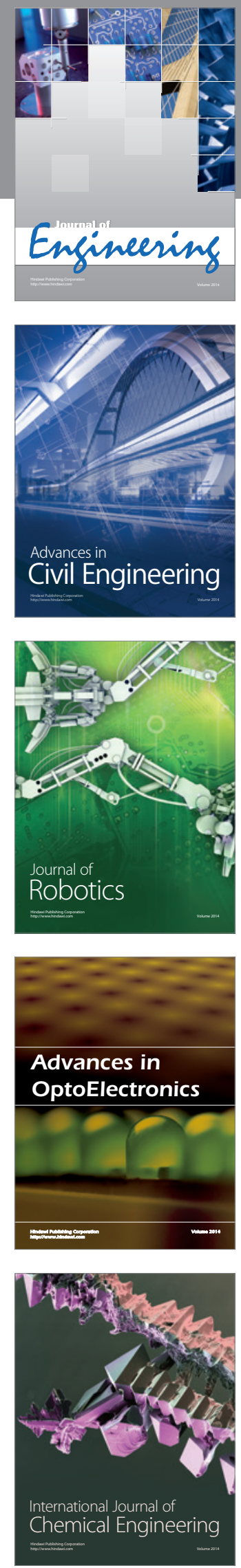

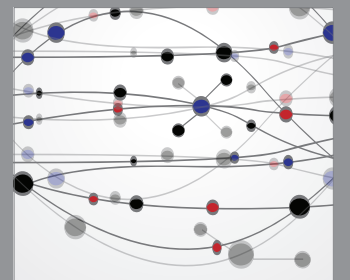

The Scientific World Journal
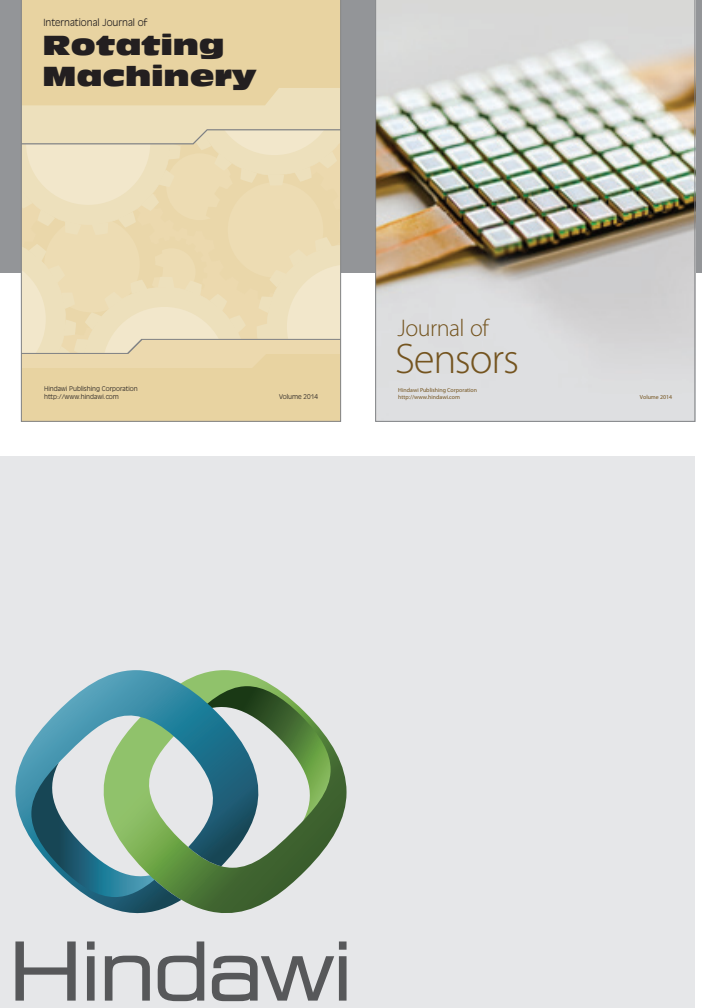

Submit your manuscripts at http://www.hindawi.com
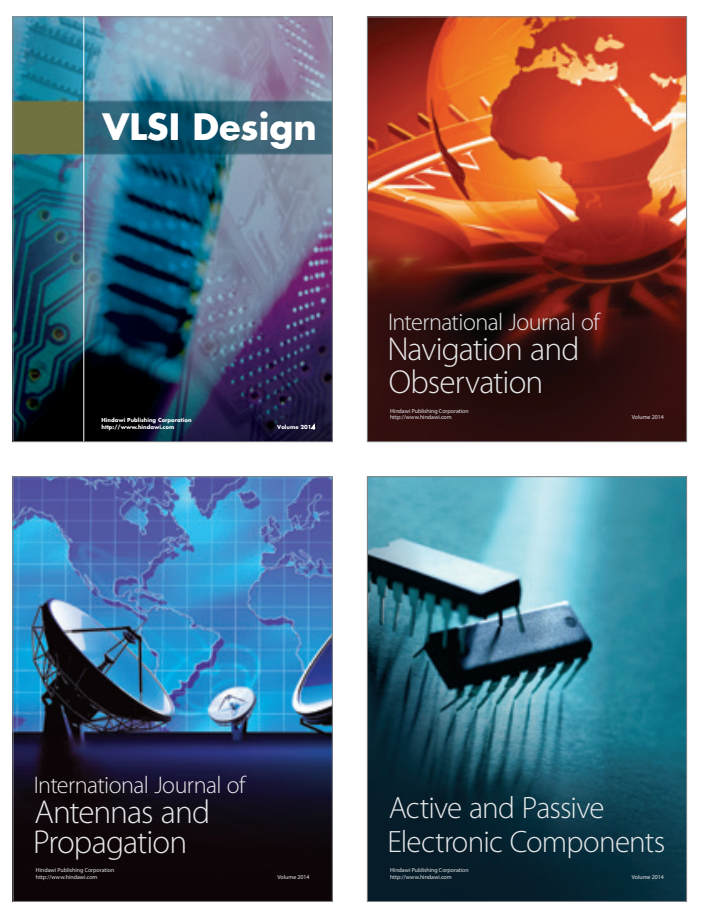
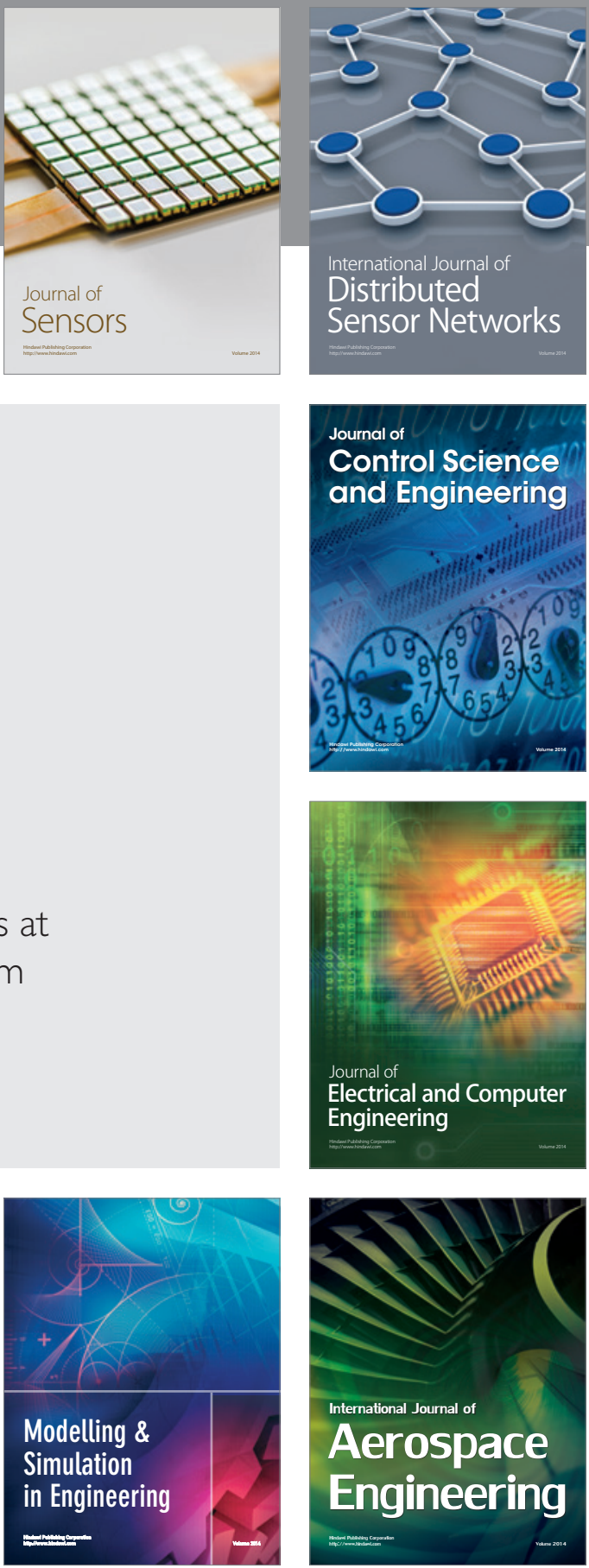

Journal of

Control Science

and Engineering
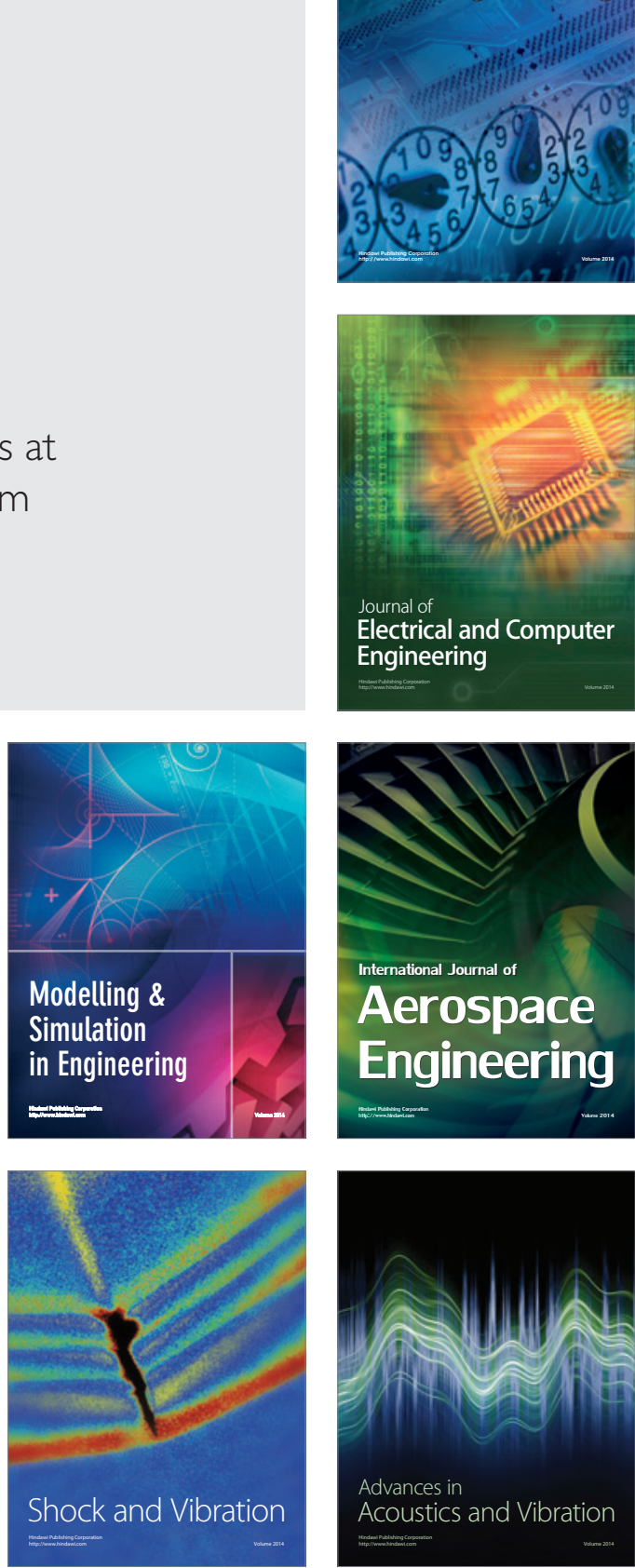